\title{
Contemporary Non-hormonal Therapies for the Management of Vasomotor Symptoms Associated with Menopause: A Literature Review
}

\author{
Sabrina Sahni, ${ }^{1}$ Angie Lobo-Romero ${ }^{2}$ and Taryn Smith ${ }^{3}$ \\ 1. Division of General Internal Medicine, Jacoby Center for Breast Health, Mayo Clinic, Jacksonville, FL, USA; 2. Division of General Internal \\ Medicine, Women's Health, Mayo Clinic Rochester, MN, USA; 3. Division of General Internal Medicine, Women's Health, Mayo Clinic, \\ Jacksonville, FL, USA
}

DOI: https://doi.org/10.17925/EE.2021.17.2.133

$\mathrm{N}$ early $75 \%$ of all menopausal women experience bothersome vasomotor symptoms including hot flushes and night sweats. Yet vasomotor symptoms continue to be an undertreated and underdiagnosed symptom of menopause which can negatively affect a woman's overall quality of life. While hormone therapy has been widely utilized to ameliorate hot flushes, not all women are candidates for use, especially those with increased risk of cardiovascular disease, thromboembolic disease, and/or women at an increased risk of certain hormone-dependent cancers. The current literature provides strong evidence for non-hormonal therapies in women who experience vasomotor symptoms. This article reviews the evidence for the use of non-hormonal pharmacologic therapies for the treatment of menopausal symptoms including antidepressants, gabapentinoids, clonidine and anticholinergics. We also review data on emerging therapies including the latest evidence on neurokinin-1 and -3 antagonists. These therapies should be considered when hormonal options are contraindicated and/or not preferred by the patient. While there are many options available, clinicians should individualize therapy based on the patient's needs and goals while mitigating bothersome side effects.

\section{Keywords}

Vasomotor symptoms, menopause, hormone therapy, non-hormonal therapy, hot flushes

Financial disclosure: Sabrina Sahni, Angie Lobo-Romero and Taryn Smith have no financia or non-financial relationships or activities to declare in relation to this article.

Review process: Double-blind peer review.

Compliance with ethics: This study involves a review of the literature and did not involve any studies with human or animal subjects performed by any of the authors.

Data availability: Data sharing is not applicable to this article as no datasets were generated or analysed during the writing of this article.

Authorship: The named authors meet the Internationa Committee of Medical Journal Editors (ICMJE) criteria for authorship of this manuscript, take responsibility for the integrity of the work as a whole and have given final approval for the version to be published.

Access: This article is freely accessible at

touchENDOCRINOLOGY.com (c) Touch Medical Media 2021

Received: 27 April 2021

Accepted: 28 June 2021

Published online: 13 October 2021

Citation: touchREVIEWS in Endocrinology. 2021; 17(2):133-7

Corresponding author: Taryn Smith, Division General Internal Medicine, Women's Health Specialty Clinic Mayo Clinic, Jacksonville, Florida, 4500 San Pablo Rd S, Jacksonville, FL 32224, USA. E: Smith.taryn@mayo.edu

Support: No funding was received in

the publication of this article
Nearly $75 \%$ of all menopausal women experience bothersome vasomotor symptoms, including hot flushes and night sweats.' Declining levels of estrogen during menopause lead to a narrowing of the central thermal regulatory neutral tone. Minute changes in core body temperature, as little as $0.8 \pm 0.99^{\circ} \mathrm{C}$, can often trigger sweating and flushing in both perimenopausal and postmenopausal women. ${ }^{1}$ Vasomotor symptoms can also be accompanied by mood changes, worsening anxiety or depression and 'brain fog'. As a result, these symptoms have also been shown to negatively affect work productivity, interpersonal relationships, sleep quality and overall quality of life in menopausal women. ${ }^{2}$ When left untreated, vasomotor symptoms have been shown to substantially increase healthcare resource utilization as well as direct and indirect healthcare-related cost in the United States. ${ }^{3,4}$ Menopausal hormone therapy is considered a standard treatment for bothersome vasomotor symptoms. ${ }^{5}$ For the average healthy postmenopausal woman, hormone therapy is considered a safe and effective treatment of bothersome vasomotor symptoms when initiated within 10 years of menopause or under the age of 60 years. ${ }^{4,5}$ In addition to quality of life benefits, menopausal hormone therapy is a recognized treatment for reducing the risk of fractures associated with osteoporosis. Recent publications have also addressed the cardiovascular benefits of menopausal hormone therapy, especially in women who undergo early menopause. ${ }^{6}$

Despite the well documented benefits of menopausal hormone therapy, utilization of US Food and Drug Administration (FDA)-approved therapies has declined in eligible women due to perceived safety concerns. ${ }^{5}$ Given the burden that vasomotor symptoms may have on an individual woman, non-hormonal treatments should be considered for the management of bothersome vasomotor symptoms when hormonal options are contraindicated or not preferred by the patient. In addition, women with a history of estrogen-dependent cancers or those who may be at elevated risk for such, those with coronary artery disease, or those with a history of stroke or venous thromboembolism should consider these alternative options. ${ }^{7}$ This article will review the evidence for using non-hormonal pharmacologic therapies for the treatment of menopausal symptoms, including antidepressants, gabapentinoids, clonidine and anticholinergics. We will also review data on emerging therapies, including the latest evidence on neurokinin-1 and -3 antagonists. 
Table 1: Available non-hormonal pharmacologic therapies for management of vasomotor symptoms

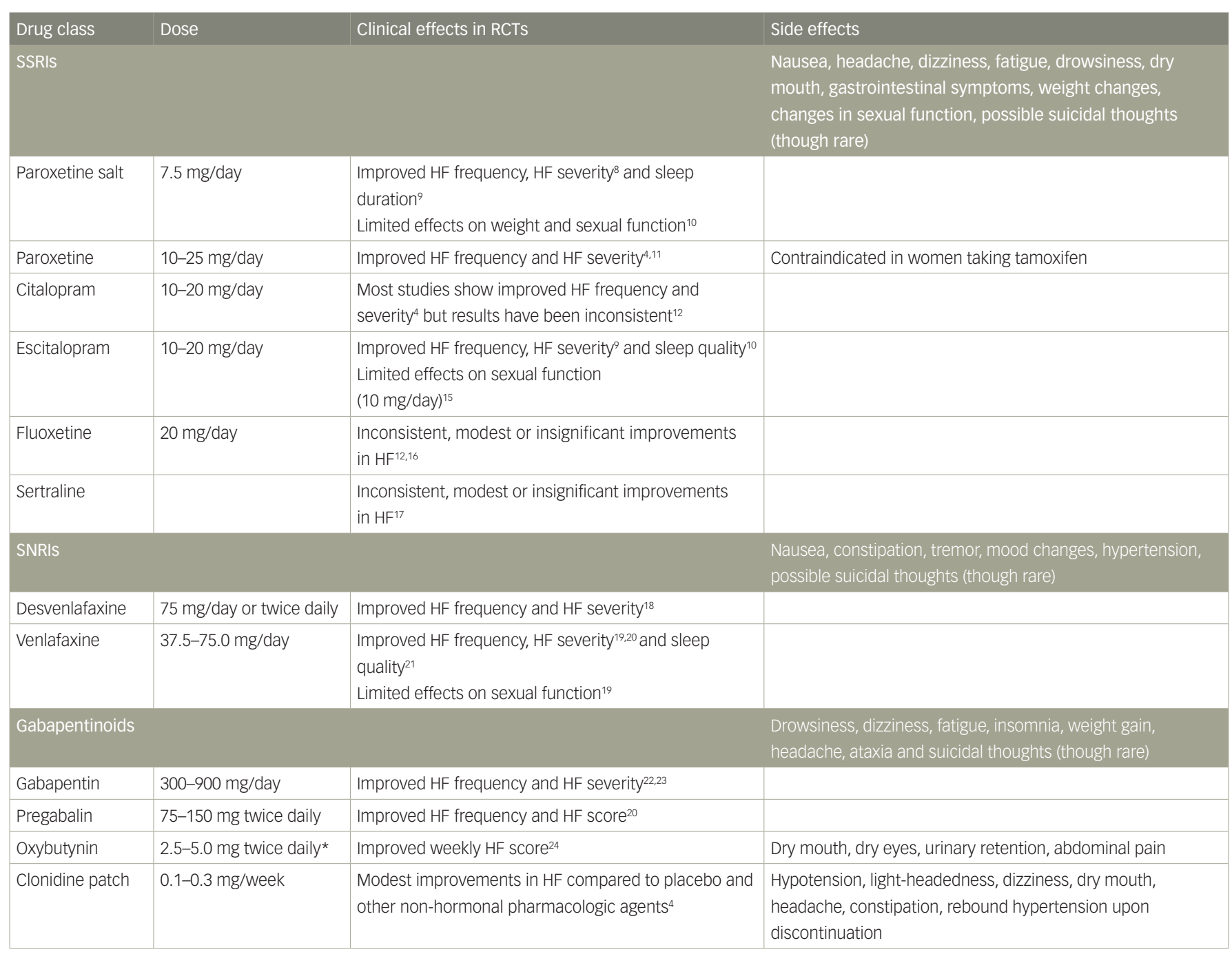

*Higher doses may be effective; however, they can lead to increased adverse anticholinergic side effects.

$\mathrm{HF}=$ hot flash; $R C T S$ = randomized controlled trials; SNRIS = serotonin norepinephrine reuptake inhibitors; SSRIS = selective serotonin reuptake inhibitors.

\section{Selective serotonin reuptake inhibitors and serotonin norepinephrine reuptake inhibitors}

In large randomized controlled trials, paroxetine, escitalopram, citalopram, desvenlafaxine and venlafaxine have been associated with statistically significant reductions in hot flush severity (Table 1). ${ }^{4,-24}$ Sertraline and fluoxetine have shown inconsistent, modest or insignificant improvements in menopausal symptoms. ${ }^{12,16,17}$

Paroxetine salt, paroxetine mesylate $(7.5 \mathrm{mg}$ daily), was shown to effectively reduce the severity and frequency of menopausal vasomotor symptoms in two randomized controlled trials of 12 and 24 weeks' duration. ${ }^{8}$ Paroxetine mesylate was well tolerated and symptom control persisted through 24 weeks of therapy. In pooled analyses, paroxetine mesylate $7.5 \mathrm{mg}$ significantly reduced night-time awakenings related to vasomotor symptoms, improved sleep duration ${ }^{9}$ and had no adverse effects on weight and sexual function. ${ }^{10}$ As strong inhibitors of cytochrome CYP2D6, paroxetine and fluoxetine limit the conversion of tamoxifen to the active metabolite endoxifen and should not be co-administered in women with a history of breast cancer who are taking tamoxifen. Currently, paroxetine mesylate $7.5 \mathrm{mg}$ is the only non-hormonal pharmacologic therapy approved by the FDA for treating moderate to severe vasomotor symptoms in postmenopausal women.
Alternatively, an 8-week, randomized, double-blind, placebo-controlled trial compared the efficacy and tolerability of oral 17-beta-estradiol $(0.5$ $\mathrm{mg} /$ day) with low dose extended release venlafaxine $(75 \mathrm{mg} /$ day) in 339 perimenopausal and postmenopausal women with greater than 2 bothersome vasomotor symptoms per day. ${ }^{25}$ The frequency of vasomotor symptoms decreased by 2.3 more per day with estradiol ( $95 \%$ confidence interval [Cl]: 1.3-3.4; $p<0.001)$ than placebo, and by 1.8 more per day with venlafaxine (95\% Cl: 0.8-2.7; $p=0.005)$ than placebo. Venlafaxine was well tolerated by study participants. A 53\% reduction in vasomotor symptom frequency was observed with estradiol, $48 \%$ with venlafaxine and $29 \%$ with placebo. This study suggests that there are only modest differences between the efficacy of oestradiol compared with venlafaxine at the studied doses. This study was limited by its short duration. Furthermore, only one dose of each study medication was used, and alternate formulations of estrogen-based menopausal therapies were not studied. A pooled analysis showed similar results suggesting that 17-beta estradiol (0.5 mg/day), venlafaxine (75 mg/day) and escitalopram (10-20mg/day) resulted in similar reductions in vasomotor symptom frequency compared with placebo after 8 weeks of therapy. ${ }^{26}$

Serotonergics are contraindicated in patients who are concomitantly using monoamine oxidase inhibitors, have a history of neuroleptic syndrome 
or are at risk of serotonin syndrome. ${ }^{1,4}$ Caution is advised in patients with polymorphisms in cytochrome P450 enzyme pathways, kidney or liver dysfunction, bipolar disorder, hyponatremia and uncontrolled hypertension..$^{1,4}$ Common side effects include nausea, fatigue, headache, dry mouth and gastrointestinal upset. ${ }^{1}$ Rarely, suicidal thoughts can be experienced when selective serotonin reuptake inhibitors (SSRIS) and serotonin norepinephrine reuptake inhibitors (SNRIS) are initiated. ${ }^{1.4}$ Effective relief of vasomotor symptoms is achieved with lower doses of SSRIS and SNRIS than what is typically needed to manage depression.

\section{Gabapentinoids}

\section{Gabapentin}

Gabapentin is a gamma-aminobutyric analogue that was initially approved in the early 1990s for the treatment of epilepsy. Since then, data has shown its efficacy in the treatment of neuropathic pain, tremors, restless leg syndrome, migraine prophylaxis and various mood disorders. ${ }^{27}$ It was not until 2000 that gabapentin was first revealed as an option for the treatment of hot flushes and hypothermia through a series of anecdotal case studies in both men and women. ${ }^{28}$ While the mechanism of action for alleviating hot flushes remains unclear, it was postulated that gabapentin's affinity for calcium channels in the hypothalamus might broaden the thermoregulatory zone, thereby decreasing the occurrence of hot flushes. ${ }^{22,27,28}$ As a result, the use of gabapentin has since been studied for the treatment of menopausal hot flushes. ${ }^{29}$

In a large meta-analysis by Shan et al., the efficacy and safety of gabapentin and pregabalin were evaluated in women suffering from vasomotor symptoms. A total of 19 randomized controlled trials and two crossover studies were included in the analysis, totalling nearly 3,500 women. Overall, gabapentin was found to reduce the frequency of hot flushes at both 4 and 12 weeks (mean difference: -1.62 [95\% Cl: -1.98 to -1.26 ], and -2.77 [95\% Cl: -4.29 to -1.24 ], respectively). ${ }^{28}$ Among the two crossover studies reported in the meta-analysis, there was no statistically significant difference between the use of gabapentin and antidepressants, namely fluoxetine and venlafaxine for the treatment of hot flushes (mean difference: -0.48 [95\% Cl: -1.53 to 0.56$]$ ). ${ }^{28}$ The daily dosage of gabapentin amongst the 21 studies varied, ranging from 300 to $2,400 \mathrm{mg}$ daily.

In a separate, smaller, meta-analysis by Yoon et al., seven randomized controlled trials were evaluated comparing the use of gabapentin to placebo for the treatment of hot flushes. ${ }^{26}$ Primary outcomes included hot flush frequency and duration in addition to a composite score, which assessed for hot flush severity. Overall, statistically significant reductions were seen in amongst all three groups and among all doses studied. Dosages ranged from 300 to $1,800 \mathrm{mg}$ per day; however, on meta-regression analysis, gabapentin was found to have prognostic value for both frequency and severity, but not hot flush duration. ${ }^{26}$ In one particular study, 59 postmenopausal women with more than seven hot flushes per day were randomized to either receive $900 \mathrm{mg} /$ day of gabapentin (divided amongst three equal doses) or placebo for a total of 12 weeks. Women who received gabapentin had a $45 \%$ reduction in the frequency of hot flushes and a 54\% reduction in composite score. ${ }^{4,27}$ Major side effects included dizziness and somnolence, which were more prevalent at week 1 and improved by week $4 .{ }^{4}$

Gabapentin should be considered an as alternative for those who have contraindications to hormone use. Given its side-effect profile, women with sleep disturbances related to vasomotor symptoms may benefit from its use. The recommended dosage of gabapentin is $900 \mathrm{mg} /$ day (divided into three equal doses of $300 \mathrm{mg}$ ), but starting doses as low as
$100 \mathrm{mg} /$ day should be considered in elderly women or those who may present with increased sensitivity. ${ }^{4}$ More data are needed to determine the lowest effective dose with the least amount of side effects.

\section{Oxybutynin}

Oxybutynin is an anticholinergic agent that is FDA approved for the treatment of overactive bladder. A common side effect of oxybutynin is decreased sweating, which has led to its use among the treatment of primary hyperhidrosis. In a study by Kim et al., the use of oxybutynin was evaluated in treating generalized hyperhidrosis in 21 postmenopausal women who had not improved on hormone therapy or other conservative treatments..$^{30}$ Women received either $5 \mathrm{mg} /$ day or $10 \mathrm{mg} /$ day, based on their symptom severity at presentation, and were followed for 3 months. All women reported improvements in hot flush frequency and severity, with improvements seen as early as day 2. Minimal side effects were reported with the most common being dry mouth.

Larger randomized controlled trials have also demonstrated benefit with oxybutynin for the treatment of hot flushes. A study by simon et al. revealed that women who took oxybutynin extended-release $15 \mathrm{mg} /$ day, for a period of 12 weeks, had significant reductions in hot flush frequency and severity compared with placebo $(p<0.007) .{ }^{31}$ Over half of the women in the treatment group reported dry mouth as a major side effect, encouraging other studies to determine if lower doses of oxybutynin could produce the same relief while mitigating the side-effect profile.

In a study by Leon-Ferre et al., 150 women, 65\% of whom had a diagnosis of breast cancer and were receiving active endocrine therapy, were randomized to oxybutynin $2.5 \mathrm{mg}$ twice daily, oxybutynin $5.0 \mathrm{mg}$ twice daily or placebo. At the end of 6 weeks, women in the higher-dose arm reported an $86 \%$ reduction in hot flush frequency, compared with a $70 \%$ reduction in the low-dose oxybutynin arm and $27 \%$ reduction in the placebo group. ${ }^{24}$ Overall side effects were mild but were more pronounced in the higher-dose group. Because of its anticholinergic properties, oxybutinin should be used with caution in elderly women who have multiple comorbidities or polypharmacy. It may otherwise be an attractive option for younger, healthy women or those who have a history of breast cancer and are currently on endocrine therapy.

\section{Clonidine}

Clonidine is an alpha-2 adrenergic agonist that is commonly used to treat hypertension. Its mechanism of action in the treatment of hot flushes is likely due to the narrowing of the thermoneutral zone by lowering levels of norepinephrine. ${ }^{32}$ While many studies demonstrate improvement with its use, ${ }^{32,33}$ results are overall modest when compared with placebo. In comparison with other agents, such as SSRIS/SNRIS and gabapentin, it is less effective. ${ }^{4}$ There are also several reported side effects including dizziness, light-headedness, hypotension and dry mouth, which make it a less desirable alternative.

\section{Emerging therapies: Neurokinin B antagonist}

Tachykinins are one of the largest families of peptides involved in neurotransmission and inflammatory processes. The three classical members of the tachykinin family are substance $P(S P)$, neurokinin A (NKA) and NKB. Tachykinin receptors have been divided into three different types, neurokinin1 (NK1), NK2 and NK3 receptors, which have preferential (but not exclusive) affinities for SP, NKA and NKB, respectively. ${ }^{34-36}$ Rance et al. hypothesized that hot flushes may be generated by specific hypothalamic neuronal pathways, which include kisseptin, neurokinin B and dynorphin (KNDy) neurons. ${ }^{37}$ The authors showed that the ablation of the KNDy neurons reduced vasodilation and partially blocked the 
effects of estrogen deficiency on thermoregulation..$^{37}$ NK3 receptor and its NKB ligand have also been implicated in the aetiology of hot flushes. ${ }^{38-40}$ Previous studies have demonstrated that NK3 antagonists rapidly reduce the number and severity of hot flushes. Furthermore, NK1 receptor desensitization attenuates cutaneous blood vessel dilation, ${ }^{39-42}$ and infusion of SP causes vasodilation and flushing of the face and neck, characteristic of postmenopausal hot flushes. ${ }^{43-45}$

\section{Neurokinin 3 receptor antagonist}

Neurokinin 3 receptor antagonists are new and emerging therapies for the treatment of moderate-to-severe menopausal vasomotor symptoms. Fezolinetant is a non-hormonal therapy currently in clinical development which acts by blocking NKB signalling and decreasing the activity of KNDy neurons. ${ }^{46}$ Depypere et al. conducted a phase lla randomized, clinical proof-of-concept trial investigating the effects of 12 weeks of fezolinetant $90 \mathrm{mg}$ twice daily compared with placebo on vasomotor symptom severity and frequency in menopausal women aged 40-65 years with moderate-to-severe vasomotor symptoms. ${ }^{47}$ Women taking fezolinetant $90 \mathrm{mg}$ twice daily experienced significant reductions in the severity and frequency of moderate to severe vasomotor symptoms compared with placebo as early as the first day of treatment.

VESTA, a 12-week, phase $1 \mathrm{lb}$, dose-ranging study, evaluated the safety and efficacy of fezolinetant (15, 30, 60 and $90 \mathrm{mg}$ twice daily, and 30, 60 and $120 \mathrm{mg}$ daily) versus placebo in women aged 40-65 with more than 50 moderate-to-severe vasomotor symptoms per week. ${ }^{48}$ At week 12 , all doses of fezolinetant resulted in a $74-87 \%$ reduction in the frequency of vasomotor symptoms compared with $55 \%$ with placebo. The severity of vasomotor symptoms improved with all doses of fezolinetant at week 4 compared with placebo. Sustained improvements in the severity of vasomotor symptoms were seen with fezolinetant at week 12 compared with placebo at the following doses: $60 \mathrm{mg}$ twice daily, $90 \mathrm{mg}$ twice daily and $60 \mathrm{mg}$ daily. Side effects were similar among all treatment doses; the most common treatment-emergent adverse events included fatigue, upper respiratory tract infection, sinusitis, headache, nausea, diarrhoea and cough. Rare but serious side effects included elevated liver function values, liver injury, retinal detachment, cholelithiasis and adjustment disorder with depressed mood.

Similarly, Prague et al. performed a 4-week, double-blind, randomized, controlled trial of an oral NK3 receptor antagonist (MLE4901; 40 mg twice daily) in women aged 40-62 years experiencing bothersome vasomotor symptoms. ${ }^{49}$ Treatment with MLE4901 resulted in a $72 \%$ reduction (95\% Cl: $-81.3,-63.3 \%$ ) reduction in hot flush frequency compared with baseline and a 51\% reduction compared with placebo $(p<0.0001)$. These effects were evident by day 3 of treatment and were unchanged throughout the study period. ${ }^{49}$ Improvements in hot flush severity, bother and interference were evident by day 3 of treatment and continued to improve throughout the 4-week study. Compared with placebo, hot flush severity improved by $31 \%(p<0.0001)$ by day 3 and by $39 \%$ by day 28 of treatment. In a separate study by Prague et al., asymptomatic rises in transaminase concentrations in a small subgroup of participants receiving MLE4901 were noted. ${ }^{41}$ No serious adverse outcomes were reported in this study. ${ }^{41}$

\section{Dual neurokinin 1 and 3 antagonist}

NT-814 is a novel, selective antagonist of both NK1 and NK3 receptors. The RELENT-1 study was a small, 14-day, randomized, controlled, phase II, dosing trial of the safety, efficacy and pharmacokinetics of NT-814 $(50,100,150$, and $300 \mathrm{mg}$ daily) versus placebo in 76 postmenopausal women with moderate-to-severe hot flushes. ${ }^{40}$ Women randomized to
$150 \mathrm{mg}$ NT-814 experienced a greater reduction in symptom frequency compared with other treatment groups. From baseline to study completion, the frequency of hot flushes reduced by $84 \%(p<0.001)$ in the $150 \mathrm{mg}$ group compared with $37 \%$ in the placebo group and $24 \%$ $(p=0.048), 59 \%(p=0.155)$ and $66 \%(p=0.022)$ in the $50 \mathrm{mg}, 100 \mathrm{mg}$ and $300 \mathrm{mg}$ groups, respectively. This pattern was similar to results reported for improvements in night-time awakenings. Given the short duration and small sample size, the study was underpowered to find consistent, statistically significant effects in the $50 \mathrm{mg}, 100 \mathrm{mg}$, and $300 \mathrm{mg}$ groups.

SWITCH-1, a 12-week, phase IIb, double-blind, randomized, controlled trial of postmenopausal women aged 40-65 years with moderate-tosevere vasomotor symptoms, was designed to ascertain optimal doses of NT-814..$^{50}$ Participants were randomized to placebo or NT-814 (40 mg, $80 \mathrm{mg}, 120 \mathrm{mg}$ or $160 \mathrm{mg}$ daily). The frequency of vasomotor symptoms was reduced in all groups with the greatest mean reductions from baseline to 12 weeks observed in the $120 \mathrm{mg}(7.8 ; \mathrm{p}=0.009)$ and $160 \mathrm{mg}$ (6.6; $p=0.109$ ) treatment groups. Improvements were observed as early as the first week of treatment. Statistically significant improvements in mood, sleep and menopause-specific quality of life were observed in the NT-814 $120 \mathrm{mg}$ and $160 \mathrm{mg}$ groups. No serious treatment-emergent adverse events were reported..$^{50}$

\section{Complementary therapies}

The use of complementary and alternative therapies for the treatment of menopausal symptoms has grown in popularity over the last several decades. Yet, despite this, concerns remain over the efficacy and safety of many of these modalities. Mind and body techniques, including paced respirations and relaxation techniques, have been shown to have little benefit on hot flush frequency and severity. ${ }^{50}$ Conversely, the use of cognitive behavioural therapy was studied in two randomized controlled trials, MENOS1 and MENOS2, ${ }^{51-52}$ and showed benefit in reducing hot flush severity, but not frequency, in patients with breast cancer. ${ }^{4}$ While more data are likely needed, the North American Menopause Society does recommend this in certain women as a risk-free therapy under the guidance of a certified provider. ${ }^{4}$

Natural products, including soy isoflavones, black cohosh and other vitamins and minerals, have been studied as treatments for vasomotor symptoms with inconsistent findings. In particular, the use of soy isoflavones has been shown to be no more effective than placebo in several randomized controlled trials. ${ }^{4}$ Similarly, black cohosh was not found to be effective in decreasing hot flush frequency in a large Cochrane review. ${ }^{53}$ Safety concerns surrounding the potential hepatotoxicity of black cohosh remain worrisome and women should be cautioned when being counselled on its use.

Lastly, acupuncture has become an attractive option for women who choose not to use medications or supplements. Acupuncture is a form of Chinese medicine, in which, small needles are inserted into specific points in the skin in order to achieve balance and energy throughout the body. ${ }^{4}$ To date, studies show that acupuncture alone is superior to placebo in reducing hot flush symptoms; however, when compared with sham acupuncture there were no significant differences noted. ${ }^{4}$ While acupuncture may be helpful in certain settings, its routine use for the treatment and management of hot flushes is not recommended.

\section{Conclusion}

Vasomotor symptoms continue to be an undertreated and underdiagnosed symptom of menopause, and can negatively affect a woman's overall quality of life. While hormone therapy has been widely 
utilized to ameliorate hot flushes, not all women are candidates for its use, especially those with increased risk of cardiovascular disease, thromboembolic disease and/or women at an increased risk of certain hormone-dependent cancers. In these women, non-hormonal options may be appropriate to treat bothersome symptoms. Newer, emerging therapies, such as neurokinin 3 receptor antagonists, show promising effects in reduction of vasomotor symptoms and may serve as an alternative option with fewer intolerable side effects. The current literature provides strong evidence for non-hormonal therapies in women who experience vasomotor symptoms and should be considered when hormonal options are contraindicated and/or not preferred by the patient. While there are many options available, clinicians should ultimately individualize therapy based on the patient's needs and goals while mitigating bothersome side effects. $\square$
1. Pinkerton JV, Santen RJ. Managing vasomotor symptoms in women after cancer. Climacteric. 2019;22:544-52.

2. Mirkin S, Graham S, Revicki DA, et al. Relationship between vasomotor symptom improvements and quality of life and sleep outcomes in menopausal women treated with oral, combined 17/-estradiol/progesterone. Menopause. 2019;26:637-42.

3. Sarrel P, Portman D, Lefebvre P, et al. Incremental direct and indirect costs of untreated vasomotor symptoms. Menopause. 2015;22:260-6.

4. Nonhormonal management of menopause-associated vasomotor symptoms: 2015 position statement of The North American Menopause Society. Menopause. 2015:22:115-72.

5. Smith T, Sahni S, Thacker HL. Postmenopausal hormone therapy-local and systemic: a pharmacologic perspective. $J$ Clin Pharmacol. 2020;60(Suppl. 2):S74-85.

6. El Khoudary SR, Aggarwal B, Beckie TM, et al. Menopause transition and cardiovascular disease risk: implications for timing of early prevention: a scientific statement from the American Heart Association. Circulation. 2020;142:e506-32.

. Pinkerton JV. Hormone therapy for postmenopausal women N Engl J Med. 2020;382:446-55

8. Simon JA, Portman DJ, Kaunitz AM, et al. Low-dose paroxetine $7.5 \mathrm{mg}$ for menopausal vasomotor symptoms: two randomized controlled trials. Menopause. 2013;20:1027-35.

9. Pinkerton JV, Joffe H, Kazempour K, et al. Low-dose paroxetine (7.5 mg) improves sleep in women with vasomotor symptoms associated with menopause. Menopause. 2015;22:50-8.

10. Portman DJ, Kaunitz AM, Kazempour K , et al. Effects of low-dose paroxetine $7.5 \mathrm{mg}$ on weight and sexual function during treatment of vasomotor symptoms associated with during treatment of vasomotor symptoms asso

11. Stearns V, Beebe KL, Iyengar M, Dube E. Paroxetine controlled release in the treatment of menopausal hot flushes: a randomized controlled trial. JAMA. 2003;289:2827-34.

12. Suvanto-Luukkonen $E$, Koivunen $R$, Sundström $H$, et al. Citalopram and fluoxetine in the treatment of postmenopausal symptoms: A prospective, randomized, 9-month, placebo-
controlled, double-blind study. Menopause. 2005;12:18-26.

13. Freeman EW, Guthrie KA, Caan B, et al. Efficacy of escitalopram for hot flushes in healthy menopausal women: a randomized controlled trial. JAMA. 2011;305:267-74.

14. Ensrud KE, Joffe $H$, Guthrie KA, et al. Effect of escitalopram on insomnia symptoms and subjective sleep quality in healthy perimenopausal and postmenopausal women with hot flushes: a randomized controlled trial. Menopause. 2012;19:848-55.

15. Reed SD, Guthrie KA, Joffe $\mathrm{H}$, et al. Sexual function in Reed SD, Guthrie KA, Joffe $\mathrm{H}$, et al. Sexual function in
nondepressed women using escitalopram for vasomotor symptoms: a randomized controlled trial. Obstet Gynecol. 2012;119:527-38.

16. Loprinzi CL, Sloan JA, Perez EA, et al. Phase III evaluation of fluoxetine for treatment of hot flashes. J Clin Oncol. 2002;20:1578-83

17. Grady D, Cohen B, Tice J, et al. Ineffectiveness of sertraline for treatment of menopausal hot flushes: a randomized controlled trial. Obstet Gynecol. 2007;109:823-30.

18. Pinkerton JV, Constantine G, Hwang E, Cheng RF」. Desvenlafaxine compared with placebo for treatment of menopausal vasomotor symptoms: a 12-week, multicenter, parallel-group, randomized, double-blind, placebo-controlled paralle-group, randomized, double-blind,
efficacy trial. Menopause. 2013:20:28-37.

19. Reed $S D$, Mitchell $C M$, Joffe $H$, et al. Sexual function in women on oestradiol or venlafaxine for hot flushes: a randomized controlled trial. Obstet Gynecol. 2014:124:233-41.

20. Loprinzi CL, Qin R, Baclueva EP, et al. Phase III, randomized, double-blind, placebo-controlled evaluation of pregabalin for alleviating hot flushes, N07C1. J Clin Oncol. 2010;28:641-7.

21. Ensrud KE, Guthrie KA, Hohensee C, et al. Effects of oestradio and venlafaxine on insomnia symptoms and sleep quality in women with hot flushes. Sleep. 2015;38:97-108.

22. Yoon SH, Lee JY, Lee C, et al. Gabapentin for the treatment of hot flushes in menopause: a meta-analysis. Menopause. 2020;27:485-93.

23. Shan D, Zou L, Liu X, et al. Efficacy and safety of gabapentin and pregabalin in patients with vasomotor symptoms: a systematic review and meta-analysis. Am J Obstet Gynecol. systematic review
2020:222:564-79.

24. Leon-Ferre RA, Novotny PJ, Wolfe EG, et al. Oxybutynin vs placebo for hot flashes in women with or without breast cance a randomized, double-blind clinical trial (ACCRU SC-1603) JNCl Cancer Spectr. 2020;4:pkz088.

25. Joffe H, Guthrie KA, LaCroix AZ, et al. Low-dose estradiol and the serotonin-norepinephrine reuptake inhibitor venlafaxine for vasomotor symptoms: a randomized clinical trial. JAMA Intern Med. 2014;174:1058-66

26. Guthrie KA, Lacroix AZ, Ensrud KE, et al. Pooled analysis of six pharmacologic and nonpharmacologic interventions for vasomotor symptoms. Obstet Gynecol. 2015;126:413-22.

27. Guttuso T, Kurlan R, McDermott MP, Kieburtz K. Gabapentin's effects on hot flashes in postmenopausal women: a randomized controlled trial. Obstet Gynecol. 2003:101:337-45.

28. Guttuso TJ. Gabapentin's effects on hot flashes and hypothermia. Neurology 2000:54:2161-3.

29. Brown JN, Wright BR. Use of gabapentin in patients experiencing hot flashes. Pharmacotherapy. 2009;29:74-81.

30. Kim WO, Kil HK, Yoon KB, Yoo JH. Treatment of generalized hyperhidrosis with oxybutynin in postmenopausal patients Acta Derm Venereol. 2010;90:291-3.

31. Simon JA, Gaines T, LaGuardia KD, et al. Extended-release oxybutynin therapy for vasomotor symptoms in women: a randomized clinical trial. Menopause. 2016;23:1214-21.

32. Freedman RR. Menopausal hot flashes: mechanisms, endocrinology, treatment. I Steroid Biochem Mol Biol. 2014;142:115-20

33. Laufer LR, Erlik Y, Meldrum DR, Judd HL. Effect of clonidine on hot flashes in postmenopausal women. Obstet Gynecol. 1982;60:583-6.

34. Garcia-Recio S, Gascón P. Biological and pharmacological aspects of the NK1-receptor. Biomed Res Int. 2015;2015:495704.

35. Pennefather JN, Lecci A, Candenas ML, et al. Tachykinins and tachykinin receptors: a growing family. Life Sci. 2004;74:1445-63.

36. Page NM. New challenges in the study of the mammalia tachykinins. Peptides. 2005;26:1356-8

37. Rance NE, Dacks PA, Mittelman-Smith MA, et al. Modulation of body temperature and LH secretion by hypothalamic KNDy (kisspeptin, neurokinin B and dynorphin) neurons: novel hypothesis on the mechanism of hot flushes. Front Neuroendocrinol. 2013;34:211-27.

38. Rometo AM, Krajewski SJ, Voytko ML, Rance NE. Hypertrophy and increased kisspeptin gene expression in the hypothalamic infundibular nucleus of postmenopausal women and ovariectomized monkeys. J Clin Endocrinol Metab. 2007:92:2744-50.

39. Rance NE, Young WS. Hypertrophy and increased gene expression of neurons containing neurokinin-B and substance-P messenger ribonucleic acids in the hypothalami of postmenopausal women. Endocrinology. 1991;128:2239-74.

40. Trower M, Anderson RA, Ballantyne E, et al. Effects of NT-814, a dual neurokinin 1 and 3 receptor antagonist, on vasomotor symptoms in postmenopausal women: a placebo-controlled randomized trial. Menopause. 2020;27:498-505

41. Prague JK, Roberts RE, Comninos AN, et al. Neurokinin 3 receptor antagonism as a novel treatment for menopausal hot flushes: a phase 2 randomised, double-blind, placebocontrolled trial. Lancet. 2017;389:1809-20

42. EU Clinical Trials Register. Pilot/Phase Ila Trial to Investigate the Effect of ESN364 in Early Postmenopausal Women Suffering From Hot Flashes. (EudraCT number: 2015-00257820). Available at: www.clinicaltrialsregister.eu/ctr-search/ 20). Avallable at: wWw.clinicaltrialsregister.eu/ctr-search
trial/2015-002578-20/results (accessed 16 July 2021).

trial/2015-002578-20/results (accessed 16 July 2021).
3. Santoro N, Waldbaum A, Lederman S, et al. Effect of the neurokinin 3 receptor antagonist fezolinetant on patientreported outcomes in postmenopausal women with vasomoto symptoms: results of a randomized, placebo-controlled, double-blind, dose-ranging study (VESTA). Menopause. 2020;27:1350-6.

44. Wong BJ, Minson CT. Neurokinin-1 receptor desensitization attenuates cutaneous active vasodilatation in humans. J Physiol. 2006;577:1043-51.

45. De Muckadell OBS, Aggestrup S, Stentoft P. Flushing and plasma substance $P$ concentration during infusion of synthetic substance P in normal man. Scand I Gastroenterol. synthetic substance

46. Hoveyda HR, Fraser GL, Dutheuil G, et al. Optimization of novel antagonists to the neurokinin-3 receptor for the treatment of sex-hormone disorders (Part II). ACS Med Chem Lett 2015;6:736-40

47. Depypere $H$, Timmerman D, Donders $G$, et al. Treatment of menopausal vasomotor symptoms with fezolinetant, a neurokinin 3 receptor antagonist: a phase 2a trial. J Clin Endocrinol Metab. 2019;104:5893-905.

48. Fraser $\mathrm{GL}$, Lederman $\mathrm{S}$, Waldbaum $\mathrm{A}$, et al. A phase $2 \mathrm{~b}$, randomized, placebo-controlled, double-blind, dose-ranging study of the neurokinin 3 receptor antagonist fezolinetant for vasomotor symptoms associated with menopause. Menopause. 2020;27:389-92.

49. Prague JK, Roberts RE, Comninos AN, et al. Neurokinin 3 receptor antagonism rapidly improves vasomotor symptoms with sustained duration of action. Menopause 2018:25:862-9.

50. Simon J, Anderson RA, Ballantyne E, et al. OR11-03 NT-814, a non-hormonal dual neurokinin 1,3 receptor antagonist markedly improves vasomotor symptoms in post-menopausal women; results of a randomised, double-blind, placebocontrolled, dose-finding study (SWITCH-1). J Endocr Soc 2020;4(Suppl. 1):OR11-03

51. Mann E, Smith M, Hellier J, Hunter MS. A randomised controlled trial of a cognitive behavioural intervention for women who have menopausal symptoms following breast cancer treatment (MENOS 1): Trial protocol. BMC Cancer. 2011;11:44.

52. Ayers B, Smith M, Hellier J, et al. Effectiveness of group and self-help cognitive behavior therapy in reducing problematic menopausal hot flushes and night sweats (MENOS 2): a randomized controlled trial. Menopause. 2012;19:749-59.

53. Leach MJ, Moore V. Black cohosh (Cimicifuga spp.) for menopausal symptoms. Cochrane Database syst Rev. 2012:2012:CD007244. 